\title{
Association of HMGB1, S100A12 and IL-17A Expression with Immunoglobulin-Resistant in Kawasaki Disease and the Comparisons Between Different Adjunctive Therapeutic Approaches
}

\section{Yang Zhou}

Wuhan Childrens Hospital: Wuhan Women and Children Medical Care Center

Liu Pan

wuhan wuchang hospital

You-jun Yang

Wuhan Childrens Hospital: Wuhan Women and Children Medical Care Center

Shi-yu Li

Wuhan Childrens Hospital: Wuhan Women and Children Medical Care Center

Wei Yin

Wuhan Childrens Hospital: Wuhan Women and Children Medical Care Center

Fan Liu

Wuhan Childrens Hospital: Wuhan Women and Children Medical Care Center

yan ding ( $\square$ dingyanmx@163.com )

Wuhan Childrens Hospital: Wuhan Women and Children Medical Care Center https://orcid.org/00000001-9157-1324

Research article

Keywords: Kawasaki disease, IVIG-resistant, laboratory parameters, clinical symptoms, adjunctive therapy.

Posted Date: January 10th, 2022

DOI: https://doi.org/10.21203/rs.3.rs-1163979/v1

License: (c) (1) This work is licensed under a Creative Commons Attribution 4.0 International License.

Read Full License 


\section{Abstract}

Objective: The DAMPs such as HMGB1, S100A12 and IL-17A have been reported to predict poor response to IVIG. The aim of this study was to analyze the role of HMGB1,S100A12 and IL-17A in the detection of inflammation in KD patients with IVIG-resistant, and to investigate the value of different adjunctive therapy.

Method: This study enrolled 126 patients diagnosed with KD, as well as age-matched 16 febrile control subjects. The demographic or clinical data, laboratory parameter and blood sample were collected. Various laboratory parameters as predictive factors for IVIG-resistant were calculated. And the serum levels of IL-17A and mRNA expression levels of HMGB1 and S100A12 were tested in all patients. For patients with acute KD in IVIG-resistant, we studied the levels of laboratory variables when using of IVIG retreatment, methylprednisolone, infliximab for children patients.

Result: The variance of laboratory parameters between the febrile control group and KD group were analyzed. Regarding laboratory parameters, $K D$ individuals were found to have lower levels of $L \%, P A$, CD4+, CD8+ and higher levels of WBC, N\%, CRP, ESR, NT-proBNP, ALT, CD4+/CD8+ $(P<0.05$ or $P<0.01)$. For KD group, the 53 IVIG-resistant patients had significantly higher levels of blood S100A12, HMGB1, serum IL-17A levels And N\%, CRP, NT-pro BNP, TBIL, ALT, AST and lower levels of $L \%, P L T(P<0.05$ or $P<0.01)$ in comparison to the IVIG-responsive patients. For patients with acute KD in IVIG-resistant, after initial IVIGtreatment, the adjunctive therapy of IVIG, methyl prednisolone or infliximab were used, the inflammatory symptoms and laboratory inflammatory markers were improved when treated with those drugs.

Conclusion: IVIG-resistant was associated with higher levels of HMGB1, S100A12, IL-17A, CRP, NT-pro BNP, TBIL, ALT, AST and lower levels of L\%, PLT before IVIG, especially when combined, were useful predictors for IVIG-resistant in KD. In addition, the adjunctive therapy of methylprednisolone and infliximab showed more effective in relief clinical symptoms than IVIG retreatment.

\section{Introduction}

Kawasaki disease (KD), an acute febrile illness that causes systemic vasculitis in children[1], is now the most common cause of acquired heart disease in developed countries[2] and leads to serious coronary complications[3, 4]. Timely infusion of high-dose intravenous immunoglobulin (IVIG) and aspirin is the standard treatment for KD[5], has declined the incidence of coronary artery lesions(CALs)[1, 6]. However, $10-20 \%$ of KD patients develop recurrent or persistent fever after the end of initial IVIG treatment are named IVIG resistant[7, 8], and these patients are at higher risk of CAL and acquired cardiac complications. Therefore, identifying some biomarkers of patients who are likely to be IVIG-resistant would allow the use of adjunctive therapies to eliminate the inflammatory process early in the course of their illness when prevention of coronary artery damage.

The damage associated molecular patterns(DAMPs) such as $\mathrm{S} 100$ calcium-binding protein A12 (S100A12) and high-mobility group protein B1 (HMGB1) have been reported to be a sensitive indicator for 
disease activity and inflammation in many inflammatory disorders, and may be closely associated with the development of CALs in $\operatorname{KD}[9,10]$.These markers are released from stressed cells and binding with receptor for advanced glycation end products (RAGE) or Toll-like receptors (TLRs) to activate the NF-KB pathway to produce endothelial damage and lead to CAL development in KD patients[11]. In addition, recent studies have found that up-regulation of T helper (Th) 17 cells and down-regulation Treg cells are associated with many autoimmune diseases. T cell-derived cytokine includes a 6-member ligand (IL17A IL-17F)[12]. IL-17A, tumor necrosis factor (TNF-a) and IL-6 secreted by Th17 cells have proinflammatory properties, which can collectively mobilize, recruit and activate neutrophils, thus mediating inflammation of the tissues[13, 14].Plasma levels of Th17- and Treg-related cytokines Interleukin-17A (IL-17A) are highly expressed in KD[13]. Recently, there are only a handful of studies examing the expression of IL-17A, HMGB1 and S100A12 to identify the responsiveness of IVIG treatment in KD patients.

IVIG resistance is a high risk factor for coronary involvement[15]. Therefore, intensification of identification and early pre-judgment of non-response to IVIG has an important guiding role in managing KD[16]. Higher level of C-reactive protein (CRP), neutrophils $(\mathrm{N})^{[17]}$, aspartate aminotransferase (AST)[18, 19], N-terminal pro-brain natriuretic peptide (NT-pro BNP)[20] and lower level of platelet(PLT) counts[21, 22] have been known as the risk factors for IVIG non-response KD. Thus, IVIG-resistant patients are associated with severe inflammatory reactions, adjunctive treatment are needed to interfere the inflammatory process[23]. Recently, although several adjunctive therapies such as IVIG retreatment, corticosteroids, infliximab and Others are available, comparative robust data on which to IVIG-resistant treatment decisions are scarce[24]. IVIG appears to have a wide range of anti-inflammatory effect, and possible mechanisms of action include regulation of cytokine production, increased regulation of T-cell activity, neutralization of toxins and so on[1,25]. Methylprednisolone attributes to suppression of persistent vascular inflammation. Infliximab (TNF-a blockade) binds specifically to human TNF-a and is indicated for the treatment of immune-modulated inflammatory disorders. Thus, We summarized the experience with three different adjunctive therapies of IVIG retreatment, methylprednisolone and infliximab from clinical trials in Wuhan Children's Hospital to guide the choice of Optimal therapeutic agents to clinicians for the children with IVIG resistance.

In this study, identification of predictive biomarkers to segregate IVIG response and IVIG resistance remains an interesting subject of investigation. Therefore, we measured some laboratory characteristics as potential risk factors for IVIG resistance KD. And we propose the hypothesis that these DAMPs(HMGB1,S100A12) and IL-17A in children KD are at high risk for IVIG resistance. Also, different adjunctive therapies will show their special effect in improving clinical symptoms or inflammatory symptoms.

\section{Materials And Methods Patients Studied}


We studied 126 patients who were diagnosed according to the criteria established by the American Heart Association (AHA) in Wuhan Children's Hospital[1]. The diagnosis of classic KD is according to the presence of fever at least 5 days and the presence of $\geq 4$ of the 5 following principal clinical criteria:

1. strawberry tongue, fissure and erythema lips

2. bilateral nonpurulent conjunctivitis

3. maculopapular or erythema multiforme-like rashes

4. redness and swelling of the hands and feet or periungual membrane desquamation

5. unilateral cervical lymphadenopathy.

We took the peripheral blood samples of all patients when they were admitted to hospital. The informed consent was obtained from all patients and guardians. Patients were classified into two groups: normal control group (NC group) and KD group. Moreover, KD group was divided into IVIG-responsive group and IVIG-resistant group. All the KD patients were treated with an initial IVIG infusion at $2 \mathrm{~g} / \mathrm{kg}[26]$. We defined IVIG-responsive as defervescence within 36 to 48 hours after the initial IVIG infusion and no recurrence(temperature $>38^{\circ} \mathrm{C}$ ), IVIG-resistant patients were defined as those who exhibited persistence of fever $\left(T>38^{\circ} \mathrm{C}\right)$ beyond 36 to $48 \mathrm{~h}[5,26]$. In our study, there were 53 IVIG-resistant patients with $\mathrm{KD}$ whose temperature were still higher than $38^{\circ} \mathrm{C}$ after 36 to $48 \mathrm{~h}$ for standard treatment of initial IVIG together with Acetylsalicylic Acid(ASA). Then 23 IVIG-resistance patients were continued with IVIG at 2g/kg, 26 IVIGresistant patients were treated with methylprednisolone $(20 \mathrm{mg} / \mathrm{kg} / \mathrm{d}$ for 3 consecutive days), and 4 patients were treated with infliximab ( $5 \mathrm{mg} / \mathrm{kg}$ intravenously over 2 hours). Laboratory indicators at admission(KD1), after initial IVIG(KD2), and at different treatment(KD3) laboratory variables were recorded.

\section{Sample laboratory collection}

All peripheral blood samples used in this study were obtained at admission. Demographic variables included Median age range, gender, pyrexia, CAL formation and IVIG-resistant. Laboratory variables included white blood cell count(WBC), N, lymphocytes(L), PLT, CRP, procalcitonin(PCT), erythrocyte sedimentation rate (ESR), serum albumin(ALB), total bilirubin(TBIL), AST, ALT, NT-proBNP, CD4+ and CD8+ T cells (\%) etc.

\section{Quantitative RT-PCR}

Whole blood the expression levels of mRNA for S100A12 and HMGB1 were measured in 73 IVIGresponsive and 53 IVIG-resistant KD patients using real-time PCR. Total RNA from Whole blood of KD patients was isolated by using RNA-Trizol (Takara, Dalian, China), and reverse transcription (RNA $\rightarrow$ CDNA) was carried out following the manufacturer's instruction of kit (Prime Script ${ }^{\text {TM }}$ RT Master Mix kit, Code: RR036A). After the template CDNA was synthesized, the amplification steps and the reaction

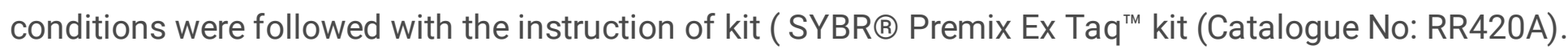
The procedure were repeated for three times and the data were analyzed using the $2^{-\Delta \Delta \mathrm{Ct}}$ method[27]. Sequences of primers for real-time PCR are included in Table 1. 
Table 1

The sequences of all primers for rt-PCR.

\begin{tabular}{|c|c|c|}
\hline Gene & primer sequence & Human $\left(5^{\prime} \rightarrow 3^{\prime}\right)$ \\
\hline \multirow[t]{2}{*}{ HMGB1 } & forward primer & 5'-TCAAAGGAGAACATCCTGGCCTGT-3' \\
\hline & reverse primer & 5'-CTGCTTGTCATCTGCAGCAGTGTT-3' \\
\hline \multirow[t]{2}{*}{ S100A12 } & forward primer & 5'-TCTAAGGGTGAGCTGAAGCAG-3' \\
\hline & reverse primer & 5'-CAATGGCTACCAGGGATATGA-3' \\
\hline \multirow[t]{2}{*}{ GAPDH } & forward primer & 5'-GGTGAAGGTCGGAGTCAACGG-3' \\
\hline & reverse primer & 5'-GGTCATGAGTCCTTCCACGATACC-3' \\
\hline
\end{tabular}

\section{serum IL-17A Levels}

We treated the level of IL-17A in human serum by the ELISA method in according to the manufacturer's instruction[27]. The sensitivity of the human IL-17A ELISA kit (Elabscience, Catalogue No: E-EL-H0105c) was $18.75 \mathrm{pg} / \mathrm{mL}$, and the intra- and inter-assay precision was below $10 \%$.

\section{Statistical Analysis}

Data are expressed as the mean \pm SD for a percentage for categorical variables. We analysed demographic, quantitative data or mRNA expression levels with Student's t-test or one-way ANOVA. SPSS version 12.0 software was used for statistical analyses. The figures in this study were generated by using GraphPad Prism 5.0 software.

\section{Results}

\section{Demographic data}

We recruited a total of 126 patients with KD $(1.85 \pm 0.20$ years old, 83 male) for this case control study (Table 2). Another 16 patients with an acute febrile infectious disease ( $2.39 \pm 0.45$ years old, 9 male) were selected as a normal control group. The acute infections among the normal control group were upper or lower respiratory tract infections or gastroenteritis. No significant difference in age or gender was found between the KD patients and the control group. Of all the participants, 32 patients (25.40\%) had CAL formation, and fifty three patients (42.06\%) were IVIG-resistant. 
Table 2

The patients with KD.

\begin{tabular}{|ll|}
\hline characteristics & Patients with KD (N=126) \\
\hline Median age range (years) & $1.85 \pm 0.20$ \\
\hline Male(n(\%)) & 65.87 \\
\hline Pyrexia & $5.81 \pm 0.26$ \\
\hline CAL formation & 32 \\
\hline IVIG resistant & 53 \\
\hline
\end{tabular}

Table 3

Laboratory characteristics of the KD group and control group

\begin{tabular}{|c|c|c|}
\hline Parameter & Control $(\mathrm{N}=16)$ & $\mathrm{KD}(\mathrm{N}=126)$ \\
\hline WBC & $11.32 \pm 1.24$ & $15.15 \pm 3.32^{\star}$ \\
\hline Neutrophil(\%) & $56.74 \pm 6.32$ & $68.08 \pm 1.50 *$ \\
\hline Lymphocyte(\%) & $34.66 \pm 6.08$ & $24.57 \pm 1.46^{*}$ \\
\hline PLT & $268.10 \pm 19.28$ & $362.30 \pm 12.85^{\star}$ \\
\hline CRP & $33.28 \pm 10.73$ & $95.50 \pm 5.54 \star \star$ \\
\hline PCT & $0.78 \pm 0.34$ & $3.02 \pm 0.86$ \\
\hline ESR & $24.13 \pm 4.84$ & $47.67 \pm 2.37^{\star \star}$ \\
\hline NT-proBNP & $316.70 \pm 32.61$ & $1042.00 \pm 129.80 *$ \\
\hline Total bilirubin & $9.31 \pm 1.02$ & $18.15 \pm 2.00$ \\
\hline Albumin & $42.34 \pm 0.82$ & $38.34 \pm 2.50$ \\
\hline ALT & $17.38 \pm 3.00$ & $63.87 \pm 7.19 *$ \\
\hline AST & $36.00 \pm 3.64$ & $64.63 \pm 13.35$ \\
\hline PA & $114.50 \pm 10.04$ & $75.36 \pm 4.36 *$ \\
\hline CD8+ & $27.12 \pm 3.94$ & $18.58 \pm 0.59 * \star$ \\
\hline CD4+ & $38.97 \pm 2.31$ & $33.42 \pm 0.88^{*}$ \\
\hline $\mathrm{CD} 4+/ \mathrm{CD} 8+$ & $1.47 \pm 0.20$ & $2.03 \pm 0.08 *$ \\
\hline
\end{tabular}


All laboratory data used in this study were obtained at admission before the initiation of therapy. The variance of laboratory parameters between the NC group and KD group were analyzed. Regarding laboratory parameters, $K D$ individuals were found to have lower levels of $L \%, P A$ and $C D 8+, C D 4+$, and higher levels of WBC, N\%, CRP, ESR, NT-pro BNP, ALT and CD4+/CD8+; these differences were statistically significant $(P<0.05$ or $P<0.01)$. There were no other statistically significant differences in indicators of PCT, TBIL, ALB and AST between the two groups (Table 3, Figure 1).

\section{Laboratory characteristics in KD group of the IVIG- responsive and the IVIG-resistant}

In the KD group, the laboratory data of the IVIG-responsive patients and the IVIG-resistant patients were obtained before IVIG-treatment. The variance of laboratory parameters between the IVIG-responsive and the IVIG-resistant were analyzed. In terms of laboratory data of KD group, patients who were IVIGresistant had higher levels of N\%, CRP, IN-proBNP, TBIL, ALT and AST prior to IVIG therapy when compared with those who were IVIG-responsive. Meanwhile, compared with IVIG-responsive patients, the IVIGresistant patients had lower levels both of L\% and PLT. PCT, ESR, ALB, CD4+, CD8+ and CD4+/CD8+ expression levels remained higher than normal value. However, there had no significant difference when compared with IVIG-responsive patients and the IVIG-resistant patients (Table 4, Figure 2). 
Table 4

Laboratory characteristics in IVIG-responsive and IVIG-resistant group

\begin{tabular}{|c|c|c|}
\hline Parameter & IVIG-responsive (N=73 ) & $\begin{array}{l}\text { IVIG-resistant } \\
(\mathrm{N}=53)\end{array}$ \\
\hline WBC & $14.39 \pm 0.6674$ & $16.19 \pm 0.9670$ \\
\hline Neutrophil(\%) & $61.30 \pm 2.112$ & $73.63 \pm 2.226$ ** \\
\hline Lymphocyte(\%) & $28.71 \pm 1.820$ & $18.87 \pm 2.174^{\star \star}$ \\
\hline PLT & $391.4 \pm 16.07$ & $322.3 \pm 19.94^{\star \star}$ \\
\hline CRP & $84.55 \pm 7.412$ & $110.6 \pm 7.957 *$ \\
\hline PCT & $2.441 \pm 1.378$ & $3.825 \pm 0.7427$ \\
\hline ESR & $44.32 \pm 2.913$ & $52.30 \pm 3.901$ \\
\hline NT-proBNP & $614.4 \pm 58.35$ & $1746 \pm 315.3^{\star \star}$ \\
\hline Total bilirubin & $13.77 \pm 2.248$ & $24.19 \pm 3.458$ ** \\
\hline Albumin & $37.54 \pm 0.5155$ & $39.45 \pm 5.917$ \\
\hline ALT & $47.23 \pm 6.292$ & $86.77 \pm 14.24^{\star \star}$ \\
\hline AST & $41.45 \pm 3.543$ & $96.57 \pm 30.99 *$ \\
\hline PA & $76.54 \pm 4.301$ & $73.73 \pm 8.569$ \\
\hline $\mathrm{CD} 8^{+}$ & $19.12 \pm 0.7727$ & $17.83 \pm 0.9165$ \\
\hline $\mathrm{CD} 4^{+}$ & $35.07 \pm 1.261$ & $33.04 \pm 1.408$ \\
\hline $\mathrm{CD} 4^{+} / \mathrm{CD}^{+}{ }^{+}$ & $2.030 \pm 0.1050$ & $2.037 \pm 0.1106$ \\
\hline
\end{tabular}

\section{Improvement of clinical symptoms in the IVIG-resistant after different drug treatments}

The recovery of clinical symptoms in the acute phase of the three groups of IVIG non-responders and changes of index recovery after discharge were as shown in Table 5. The study compared the effects of three adjunctive therapies from seven aspects: the pyrexia before starting treatment, average time of hospital stay, antipyretic time, mucosal congestion subsiding time, lymph node swelling subsiding time, redness and swelling of the hands and feet subsiding time and CAL formation before starting treatment. The antipyretic time and mucosal congestion/redness and swelling of the hands and feet subsiding time of methylprednisolone group and infliximab group were all shorter than IVIG retreatment group $(\mathrm{P}<0.05$ or 
$P<0.01)$. There had no difference between three adjunctive therapies in lymph node swelling subsiding time $(P>0.05)$. In addition, after discharge the index recovery, we found that methylprednisolone group and infliximab group were statistically significant compared with IVIG retreatment group $(P<0.05$ or $P<0.01)$.

Table 5

The recovery of clinical symptoms after different adjunctive therapies. ${ }^{*} \mathrm{P}<0.05,{ }^{*} \mathrm{P}<0.01$ compared with

IVIG retreatment group. \#P< 0.05 Methylprednisolone group VS Infliximab group. A showed index recovery within two weeks after discharge; $\mathrm{B}$ showed index recovery within two months after discharge.

\begin{tabular}{|c|c|c|c|}
\hline Item & $\begin{array}{l}\text { IVIG } \\
\text { retreatment( } \mathrm{N}=19\end{array}$ & $\begin{array}{l}\text { Methylprednisolone } \\
(\mathrm{N}=21)\end{array}$ & )$^{\text {infliximab }(\mathrm{N}=13}$ \\
\hline Pyrexia before starting treatment & $6.17 \pm 1.82$ & $5.94 \pm 1.71$ & $6.02 \pm 1.94$ \\
\hline hospital stay & $9.11 \pm 2.41$ & $8.23 \pm 2.23$ & $8.69 \pm 2.62$ \\
\hline antipyretic time & $1.21 \pm 0.80$ & $0.75 \pm 0.51^{*}$ & $0.70 \pm 0.46^{\star}$ \\
\hline Mucosal congestion subsiding time & $1.19 \pm 0.47$ & $0.82 \pm 0.39 \star \star$ & $0.83 \pm 0.35^{\star}$ \\
\hline Lymph node swelling subsiding time & $3.47 \pm 1.36$ & $2.99 \pm 1.32$ & $3.24 \pm 1.46$ \\
\hline $\begin{array}{l}\text { redness and swelling of the hands } \\
\text { and feet subsiding time }\end{array}$ & $1.98 \pm 0.56$ & $1.47 \pm 0.61^{\star \star}$ & $1.35 \pm 0.67 * \star$ \\
\hline $\begin{array}{l}\text { CAL formation before starting } \\
\text { treatment }\end{array}$ & $6 / 19$ & $6 / 21$ & $4 / 13$ \\
\hline \multicolumn{4}{|l|}{$\begin{array}{l}\text { Follow-up after discharge of index } \\
\text { recovery }\end{array}$} \\
\hline \multicolumn{4}{|l|}{ (A:two weeks; B:two months) } \\
\hline$A$ (CAL recovery) & $0 / 6$ & $1 / 6$ & $1 / 4$ \\
\hline$A(W B C)$ & $7.71 \pm 0.58$ & $7.52 \pm 0.64$ & $8.76 \pm 1.74$ \\
\hline A (Neutrophil (\%)) & $65.15 \pm 2.02$ & $60.65 \pm 2.29 * \#$ & $62.36 \pm 2.03^{\star}$ \\
\hline $\mathrm{A}(\mathrm{PLT})$ & $703.15 \pm 56.38$ & $701.57 \pm 59.87 \#$ & $789.20 \pm 72.35^{\star \star}$ \\
\hline $\mathrm{A}(\mathrm{CRP})$ & $13.63 \pm 2.36$ & $11.47 \pm 3.02^{*}$ & $10.36 \pm 2.87^{\star *}$ \\
\hline $\mathrm{B}$ (CAL recovery) & $1 / 6$ & $2 / 6$ & $1 / 4$ \\
\hline$B(W B C)$ & $5.98 \pm 0.38$ & $6.57 \pm 0.69 \star \star$ & $6.84 \pm 1.02^{\star \star}$ \\
\hline B (Neutrophil (\%)) & $57.36 \pm 2.78$ & $59.15 \pm 1.69 *$ & $54.10 \pm 3.44 * \star \#$ \\
\hline $\mathrm{B}(\mathrm{PLT})$ & $322.11 \pm 23.68$ & $299.87 \pm 30.68^{*}$ & $311.52 \pm 34.55$ \\
\hline $\mathrm{B}(\mathrm{CRP})$ & $7.22 \pm 1.98$ & $6.25 \pm 2.54 \#$ & $3.68 \pm 2.39 * *$ \\
\hline
\end{tabular}

\section{Laboratory characteristics in the IVIG-resistant after different drug treatments}


Patients who were IVIG-resistant had no statistical difference in the following laboratory data before KD1 and KD2 when different drugs treatment. In KD3, compared with IVIG retreatment and infliximab group, methylprednisolone group showed more significant lowering $\operatorname{CRP}(P<0.05)$. The other laboratory characteristics after three adjunctive therapies, there had no significantly difference $(P>0.05)($ Table 6$)$. 
Table 6

Laboratory characteristics in IVIG-resistant patients during KD1,KD2 and KD3 points of

Kawasaki disease. KD1: before the treatment of IVIG(A); KD2: the first dose of IVIG(B); KD3:the time of different drugs treatment(C). ${ }^{*} P<0.05$ compared with IVIG-retreatment group.

\section{IVIG-resistant $(\mathrm{N}=53$ )}

\begin{tabular}{|c|c|c|c|c|}
\hline & Parameter & IVIG retreatment(19) & Methylprednisolone(21) & Infliximab(13) \\
\hline & WBC & $17.03 \pm 2.04$ & $14.79 \pm 1.30$ & $14.16 \pm 1.60$ \\
\hline \multirow[t]{8}{*}{ KD1 } & Neutrophil(\%) & $71.09 \pm 4.70$ & $78.33 \pm 2.62$ & $78.48 \pm 2.30$ \\
\hline & PLT & $357.30 \pm 38.87$ & $297.40 \pm 19.70$ & $315.50 \pm 31.43$ \\
\hline & CRP & $97.43 \pm 10.08$ & $133.00 \pm 28.93$ & $132.80 \pm 15.02$ \\
\hline & ESR & $51.47 \pm 6.79$ & $54.43 \pm 6.71$ & $56.15 \pm 7.80$ \\
\hline & ALB & $52.68 \pm 16.27$ & $34.30 \pm 1.35$ & $33.98 \pm 1.74$ \\
\hline & ALT & $91.32 \pm 22.35$ & $112.6 \pm 26.56$ & $65.85 \pm 15.17$ \\
\hline & AST & $82.89 \pm 19.61$ & $92.33 \pm 19.24$ & $50.15 \pm 7.53$ \\
\hline & WBC & $14.68 \pm 1.22$ & $15.85 \pm 1.11$ & $18.57 \pm 1.30$ \\
\hline \multirow[t]{8}{*}{ KD2 } & Neutrophil(\%) & $56.44 \pm 4.14$ & $63.60 \pm 3.02$ & $68.03 \pm 4.05$ \\
\hline & PLT & $350.50 \pm 50.90$ & $399.40 \pm 44.83$ & $356.40 \pm 48.13$ \\
\hline & CRP & $75.22 \pm 12.13$ & $86.29 \pm 11.63$ & $104.10 \pm 16.82$ \\
\hline & ESR & $69.00 \pm 5.25$ & $77.52 \pm 5.43$ & $87.46 \pm 7.78$ \\
\hline & ALB & $28.26 \pm 0.84$ & $29.00 \pm 0.95$ & $27.80 \pm 0.90$ \\
\hline & ALT & $38.08 \pm 3.77$ & $41.48 \pm 6.54$ & $27.15 \pm 4.00$ \\
\hline & AST & $30.58 \pm 2.18$ & $30.05 \pm 3.64$ & $30.77 \pm 2.49$ \\
\hline & WBC & $15.85 \pm 1.41$ & $16.16 \pm 1.27$ & $15.99 \pm 1.82$ \\
\hline \multirow[t]{7}{*}{ KD3 } & Neutrophil(\%) & $59.92 \pm 4.67$ & $59.22 \pm 3.68$ & $54.55 \pm 4.94$ \\
\hline & PLT & $520.90 \pm 40.58$ & $633.20 \pm 52.49$ & $707.20 \pm 62.27$ \\
\hline & CRP & $50.63 \pm 17.80$ & $15.42 \pm 3.15^{\star}$ & $33.21 \pm 12.03$ \\
\hline & ESR & $76.16 \pm 5.03$ & $68.90 \pm 6.16$ & $77.62 \pm 7.93$ \\
\hline & ALB & $32.34 \pm 1.01$ & $30.95 \pm 1.48$ & $30.99 \pm 1.09$ \\
\hline & ALT & $32.81 \pm 8.45$ & $33.52 \pm 6.05$ & $19.31 \pm 2.45$ \\
\hline & AST & $30.95 \pm 2.91$ & $32.38 \pm 4.35$ & $28.15 \pm 3.48$ \\
\hline
\end{tabular}




\section{Detecting HMGB1 and S100A12 expression}

Comparison with KD group, the relative expression levels of HMGB1 and S100A12 mRNA were higher than NC group $(P<0.05$, Figure3A). Subsequentely, compared with IVIG-responsive group, the mRNA levels of HMGB1 and S100A12 in IVIG-resistant group were markedly elevated $(P<0.05$, Figure3B). Moreover, the Real-time PCR melt curve indicated that the primers of S100A12 and HMGB1 were specific(Figure3C-D).

\section{serum IL-17A measurements by enzyme-linked immunoassay (ELISA)}

Comparison of IL-17A serum levels in NC group and KD group, Treg-related IL-17A expression levels increased significantly in KD group $(P<0.01$, Figure4A). Also, the expression of IL-17A in IVIG-resistant group was higher than IVIG-responsive group $(P<0.01$, Figure4B).

\section{Discussion}

Kawasaki disease (KD) is an acute febrile illness associated with vasculitis of early childhood often complicated by coronary artery lesion that significantly impair the quality of life [28-30]. The study aimed to identify some reliable indicators for predicting nonresponsiveness to the first course of intravenous immunoglobulin (IVIG) in KD patients[3, 17].Developing the early judgment of IVIG-resistant in patients with KD is crucial for earlier and more effective initial therapy in patients[2].Although several predictive biomarkers and genes have been described. There have been no reliable criteria and consistent reported for identifying children most at risk of IVIG-resistant and developing CAL. In the present study, we assessed several important clinical biomarkers and HMGB1, S100A12 or IL-17A in an effort to identify predictors of a resistance to initial IVIG and choosed the best adjunctive therapies in the relief of clinical symptoms in IVIG-resistant.

Some patients with KD fail to show defervescence with initial IVIG therapy for causes remain unknown[22].Several previous studies reported that CRP, ESR, ALT, y-GT, NT-proBNP could be used for predicting resistance to IVIG therapy and patients who are at high risk for CAL[4, 31-34].The ability to predict a lack of response to IVIG before initial therapy, would allow clinicians to identify these patients because they might benefit from more aggressive treatment[35].In addition, the molecular mechanisms of IVIG-resistant patients for anti-inflammation in KD remain unclear[13]. Recent studies have found that DAMPs such as HMGB1 and S100A12 have proven to be a sensitive marker for disease activity and inflammation in numerous inflammatory disorders[36-38].Th17- and Treg-related Cytokine are associated with many autoimmune diseases, IL-17A is reported to have been associated with KD and to provoke proinflammatory responses[13,39].

KD triggers the release of HMGB1 and S100A12, which activate toll-like receptors (TLRs) and receptor for advanced glycation end products (RAGE) in the affected area, leading to an exaggerated inflammatory response and cell death[40].Th17 cells are a newly discovered T-helper cell subset associated with the pro-inflammatory stage of autoimmunity. Their differentiation requires both TGF-b combined with either 
IL-10 or IL-21 and results in the specific expression transcription factor retinoic acid-related orphan receptor ct (ROR ct), and the production of inflammatory cytokines including IL-6 and IL-17A[41]. In our study, we found that children in KD group had higher level of IL-17A when compared to the febrile control group(NC). Our laboratory data which showed that patients with KD group had lower percentages of CD4+ Tcells and CD8+ T cells when compared to the NC group. However, this increase in serum IL-17A expression was not reflected on concordant changes in peripheral blood samples of percentage CD4+ and CD8+ T cells, but the increasely CD4+/CD8+ ratio was associated with the high expression of IL-17A. Moreover, our study showed that KD was associated with higher levels of HMGB1 and S100A12. IVIGresistant was also associated with higher levels of HMGB1 and S100A12. For IVIG-resistant, Our study demonstrated the IVIG retreatment, methylprednisolone, and infliximab which had different advantages in reducing certain laboratory indicators. Compared with infliximab and IVIG retreatment group, methylprednisolone showed more

significant lowering in $\mathrm{CRP}(\mathrm{P}<0.05)$. Moreover, the three adjunctive therapies showed same effect in improving inflammation indicators.

In our study, several details deserve further attention. First, the relatively small sample size of this study might prevent some of the detected data from being statistically significant. Second, our study results need to be validated across different hospitals, different regions, different races. Lastly, IVIG-resistant was associated with higher levels of HMGB1and S100A12, but whether it exerts effects on relative TLRs or RAGE signaling pathway remains unknown. Therefore, further work to explore the relative signaling pathway of IVIG-resistant is necessary. In addition, for IVIG-resistant patients, review of relevant laboratory indicators and cardiac ultrasound for follow-up after discharge on time after three adjunctive therapies are great needed.

In conclusion, Clinical risk factors predict patient resistance to IVIG and treatment regimens to reduce the risk of developing CAL which remain controversy. Our results demonstrated that the higher levels of WBC, N\%, CRP, ESR, NT-proBNP, ALT, CD4+/CD8+ and lower levels of L\%, PA, CD8+,CD4+ showed useful for predicting in KD patients with IVIG-resistant. And HMGB1,S100A12 and serum IL-17A before the initial treatment could also predict IVIG-resistant. In addition, methylprednisolone showed better in improving clinical symptoms than the IVIG retreatment and infliximab. Further studies on the mechanism of IVIGresistant may help identify new methods to predict and treat IVIG-resistant patients in KD.

\section{Declarations}

\section{Conflicts of interest}

The authors declare that no competing interests exist.

\section{Author contributions}


PL: Performed the experiments and wrote the manuscript. YZ: Analysed the data and wrote the manuscript. FL and WY: Edited the manuscript. SYL and YJY: Interpreted the results. YD: Conceived and designed the experiments.

\section{Funding sources}

This study was supported by Hubei Province Health and Family Planning Commission of Scientific Research Project (Nos. WJ2019H313), Wuhan City Health and Family Planning Commission of clinical medical research major project (No.WX19M03).

\section{Acknowledgements}

The authors want to thanks: Wuhan City Health and Family Planning Commission for their funding; the reviewers for their precious and constructive suggestions.

\section{Availability of data and materials}

All of the material is owned by the authors and/or no permissions are required

\section{Ethics approval and consent to participate}

The study was approved by the Wuhan Children's Hospital Ethics Committee. Written informed consent was obtained from all participants in the study. All methods were carried out in accordance with relevant guidelines and regulations.

\section{Consent for publication}

Informed consent was obtained from all subjects or, if subjects are under 18, from a parent and/or legal guardian.

\section{References}

[1] McCrindle BW, Rowley AH, Newburger JW, Burns JC, Bolger AF, Gewitz M, et al. Diagnosis, Treatment, and Long-Term Management of Kawasaki Disease: A Scientific Statement for Health Professionals From the American Heart Association. CIRCULATION. 2017; 135:e927-99.

[2] Lee HY, Song MS. Predictive factors of resistance to intravenous immunoglobulin and coronary artery lesions in Kawasaki disease. Korean J Pediatr. 2016; 59:477-82.

[3] Ou-Yang MC, Kuo HC, Lin IC, Sheen JM, Huang FC, Chen CC, et al. Plasma clusterin concentrations may predict resistance to intravenous immunoglobulin in patients with Kawasaki disease.

ScientificWorldJournal. 2013; 2013:382523.

[4] Baek JY, Song MS. Meta-analysis of factors predicting resistance to intravenous immunoglobulin treatment in patients with Kawasaki disease. Korean J Pediatr. 2016; 59:80-90. 
[5] Ogata S, Ogihara Y, Nomoto K, Akiyama K, Nakahata Y, Sato K, et al. Clinical score and transcript abundance patterns identify Kawasaki disease patients who may benefit from addition of methylprednisolone. PEDIATR RES. 2009; 66:577-84.

[6] Kuo H, Wong HS, Chang W, Chen B, Wu M, Yang KD, et al. Prediction for Intravenous Immunoglobulin Resistance by Using Weighted Genetic Risk Score Identified From Genome-Wide Association Study in Kawasaki DiseaseCLINICAL PERSPECTIVE. Circulation: Cardiovascular Genetics. 2017; 10:e1625.

[7] Agarwal S, Agrawal DK. Kawasaki disease: etiopathogenesis and novel treatment strategies. EXPERT REV CLIN IMMU. 2017; 13:247-58.

[8] Shin J, Lee H, Eun L. Verification of Current Risk Scores for Kawasaki Disease in Korean Children. J KOREAN MED SCI. 2017; 32:1991.

[9] Qi Y, Gong F, Zhang Q, Xie C, Wang W, Fu S. Reverse regulation of soluble receptor for advanced glycation end products and proinflammatory factor resistin and S100A12 in Kawasaki disease. ARTHRITIS RES THER. 2012; 14:R251.

[10] Yan L, Bjork P, Butuc R, Gawdzik J, Earley J, Kim G, et al. Beneficial effects of quinoline-3carboxamide (ABR-215757) on atherosclerotic plaque morphology in S100A12 transgenic ApoE null mice. ATHEROSCLEROSIS. 2013; 228:69-79.

[11] Galeotti C, Kaveri SV, Bayry J. Molecular and immunological biomarkers to predict IVlg response. TRENDS MOL MED. 2015; 21:145-7.

[12] Maddur MS, Sharma M, Hegde P, Lacroix-Desmazes S, Kaveri SV, Bayry J. Inhibitory effect of IVIG on IL-17 production by Th17 cells is independent of anti-IL-17 antibodies in the immunoglobulin preparations. J CLIN IMMUNOL. 2013; 33 Suppl 1:S62-6.

[13] Guo MM, Tseng WN, Ko CH, Pan HM, Hsieh KS, Kuo HC. Th17- and Treg-related cytokine and mRNA expression are associated with acute and resolving Kawasaki disease. ALLERGY. 2015; 70:310-8.

[14] Jia S, Li C, Wang G, Yang J, Zu Y. The T helper type 17/regulatory T cell imbalance in patients with acute Kawasaki disease. Clinical \& Experimental Immunology. 2010; 162:131-7.

[15] Clark DE, Denby KJ, Kaufman LM, Fill MA, Piya B, Krishnaswami S, et al. Predictors of Intravenous Immunoglobulin Non-Response and Racial Disparities in Kawasaki Disease. The Pediatric Infectious Disease Journal. 2018:1.

[16] Inamo Y, Saito K, Hasegawa M, Hayashi R, Nakamura T, Abe O, et al. Effect of dalteparin, a lowmolecular-weight heparin, as adjunctive therapy in patients with Kawasaki disease: a retrospective study. BMC PEDIATR. 2014; 14:27. 
[17] Takeuchi M, Inuzuka R, Hayashi T, Shindo T, Hirata Y, Shimizu N, et al. Novel Risk Assessment Tool for Immunoglobulin Resistance in Kawasaki Disease: Application Using a Random Forest Classifier. PEDIATR INFECT DIS J. 2017; 36:821-6.

[18] Sano T, Kurotobi S, Matsuzaki K, Yamamoto T, Maki I, Miki K, et al. Prediction of non-responsiveness to standard high-dose gamma-globulin therapy in patients with acute Kawasaki disease before starting initial treatment. EUR J PEDIATR. 2007; 166:131-7.

[19] Egami K, Muta H, Ishii M, Suda K, Sugahara Y, lemura M, et al. Prediction of resistance to intravenous immunoglobulin treatment in patients with Kawasaki disease. J Pediatr. 2006; 149:237-40.

[20] Kim MK, Song MS, Kim GB. Factors Predicting Resistance to Intravenous Immunoglobulin Treatment and Coronary Artery Lesion in Patients with Kawasaki Disease: Analysis of the Korean Nationwide Multicenter Survey from 2012 to 2014. KOREAN CIRC J. 2018; 48:71.

[21] Tremoulet AH, Best BM, Song S, Wang S, Corinaldesi E, Eichenfield JR, et al. Resistance to intravenous immunoglobulin in children with Kawasaki disease. J Pediatr. 2008; 153:117-21.

[22] Tremoulet AH, Best BM, Song S, Wang S, Corinaldesi E, Eichenfield JR, et al. Resistance to Intravenous Immunoglobulin in Children with Kawasaki Disease. The Journal of Pediatrics. 2008; 153:117-21.

[23] Tremoulet AH, Jain S, Jaggi P, Jimenez-Fernandez S, Pancheri JM, Sun X, et al. Infliximab for intensification of primary therapy for Kawasaki disease: a phase 3 randomised, double-blind, placebocontrolled trial. LANCET. 2014; 383:1731-8.

[24] Shulman ST, Rowley AH. Kawasaki disease: insights into pathogenesis and approaches to treatment. NAT REV RHEUMATOL. 2015; 11:475-82.

[25] Burns JC, Franco A. The immunomodulatory effects of intravenous immunoglobulin therapy in Kawasaki disease. Expert Rev Clin Immunol. 2015; 11:819-25.

[26] Burns JC, Best BM, Mejias A, Mahony L, Fixler DE, Jafri HS, et al. Infliximab Treatment of Intravenous Immunoglobulin-Resistant Kawasaki Disease. The Journal of Pediatrics. 2008; 153:833-8.

[27] Ding Y, Liu P, Chen Z, Zhang S, Wang Y, Cai X, et al. Emodin Attenuates Lipopolysaccharide-Induced Acute Liver Injury via Inhibiting the TLR4 Signaling Pathway in vitro and in vivo. FRONT PHARMACOL. 2018; 9.

[28] Kuo HC, Liang CD, Wang CL, Yu HR, Hwang KP, Yang KD. Serum albumin level predicts initial intravenous immunoglobulin treatment failure in Kawasaki disease. ACTA PAEDIATR. 2010; 99:1578-83.

[29] Wakiguchi H, Hasegawa S, Suzuki Y, Kudo K, Ichiyama T. Relationship between T-cell HLA-DR expression and intravenous immunoglobulin treatment response in Kawasaki disease. PEDIATR RES. 
[30] Abe J, Jibiki T, Noma S, Nakajima T, Saito H, Terai M. Gene expression profiling of the effect of highdose intravenous Ig in patients with Kawasaki disease. J IMMUNOL. 2005; 174:5837-45.

[31] Nomura Y, Masuda K, Yoshinaga M, Sameshima K, Miyata K. Patients diagnosed with Kawasaki disease before the fifth day of illness have a higher risk of coronary artery aneurysm. PEDIATR INT. 2002; 44:353-7.

[32] Sittiwangkul R, Pongprot Y, Silvilairat S, Phornphutkul C. Management and outcome of intravenous gammaglobulin-resistant Kawasaki disease. Singapore Med J. 2006; 47:780-4.

[33] Wang Y, Li Z, Hu G, Hao S, Deng X, Huang M, et al. Unique Molecular Patterns Uncovered in Kawasaki Disease Patients with Elevated Serum Gamma Glutamyl Transferase Levels: Implications for Intravenous Immunoglobulin Responsiveness. PLOS ONE. 2016; 11:e167434.

[34] Sileikiene R, Kudzyte J, Jankauskas A, Labanauskas L, Rakauskiene V, Jurkiene N, et al. Rare refractory Kawasaki disease in an adolescent boy with cardiac and diffuse coronary artery involvement. Medicina (Kaunas). 2013; 49:341-5.

[35] Lee HY, Song MS. Predictive factors of resistance to intravenous immunoglobulin and coronary artery lesions in Kawasaki disease. Korean Journal of Pediatrics. 2016; 59:477.

[36] Fu S, Gong F, Xie C, Zhu W, Wang W, Shen H, et al. S100A12 on circulating endothelial cells surface in children with Kawasaki disease. PEDIATR RES. 2010; 68:165-8.

[37] Fury W, Tremoulet AH, Watson VE, Best BM, Shimizu C, Hamilton J, et al. Transcript abundance patterns in Kawasaki disease patients with intravenous immunoglobulin resistance. HUM IMMUNOL. 2010; 71:865-73.

[38] Foell D, Ichida F, Vogl T, Yu X, Chen R, Miyawaki T, et al. S100A12 (EN-RAGE) in monitoring Kawasaki disease. LANCET. 2003; 361:1270-2.

[39] Jia S, Li C, Wang G, Yang J, Zu Y. The T helper type 17/regulatory T cell imbalance in patients with acute Kawasaki disease. CLIN EXP IMMUNOL. 2010;162:131-7.

[40] Lok KZ, Basta M, Manzanero S, Arumugam TV. Intravenous immunoglobulin (IVIg) dampens neuronal toll-like receptor-mediated responses in ischemia. J Neuroinflammation. 2015; 12:73.

[41] Wu S, Zhang X, Zhou N. [Up-regulation of serum- and glucocorticoid-inducible kinase 1 (SGK1) of CD4(+) T cells is positively related to RORC and IL-17A in patients with Kawasaki disease]. Xi Bao Yu Fen Zi Mian Yi Xue Za Zhi. 2015; 31:1378-82.

\section{Figures}




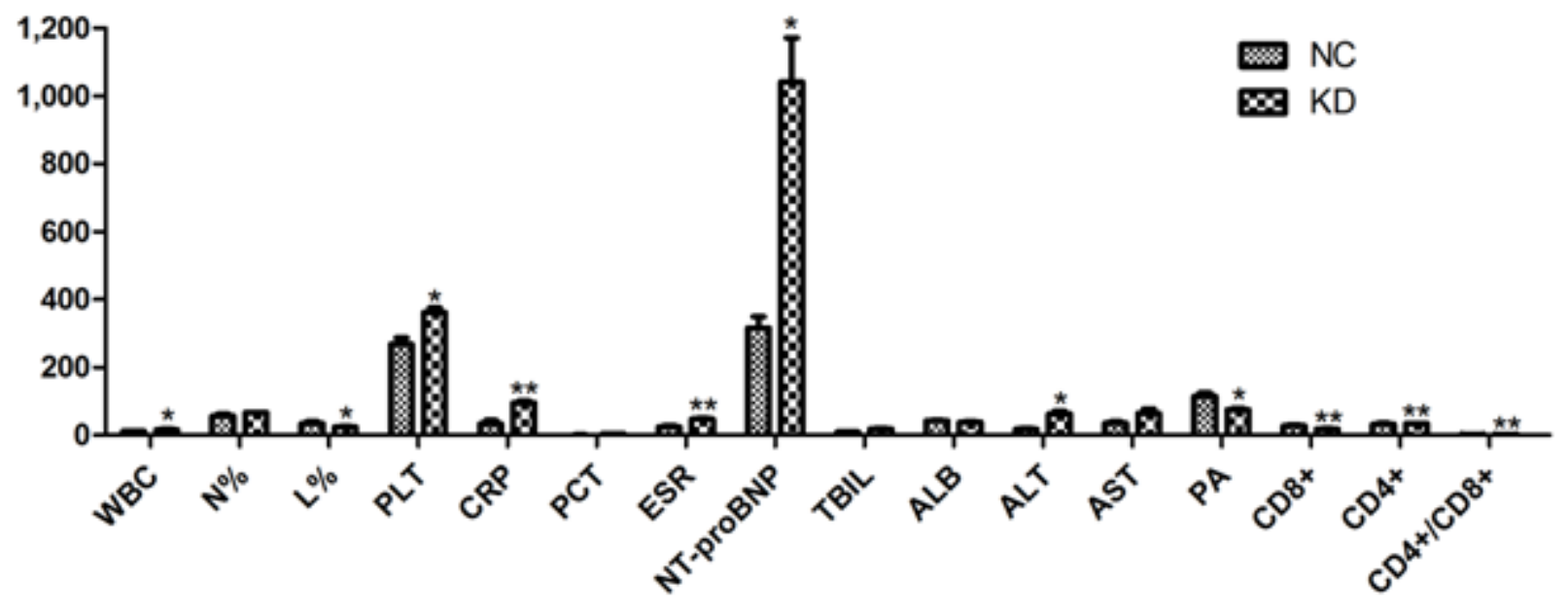

\section{Figure 1}

General analysis of whole blood isolated from children between NC group with KD group. NC group: normal control group; KD group: Kawasaki disease group. ${ }^{*} P<0.05,{ }^{*} \mathrm{P}<0.01 \mathrm{KD}$ group VS NC group.

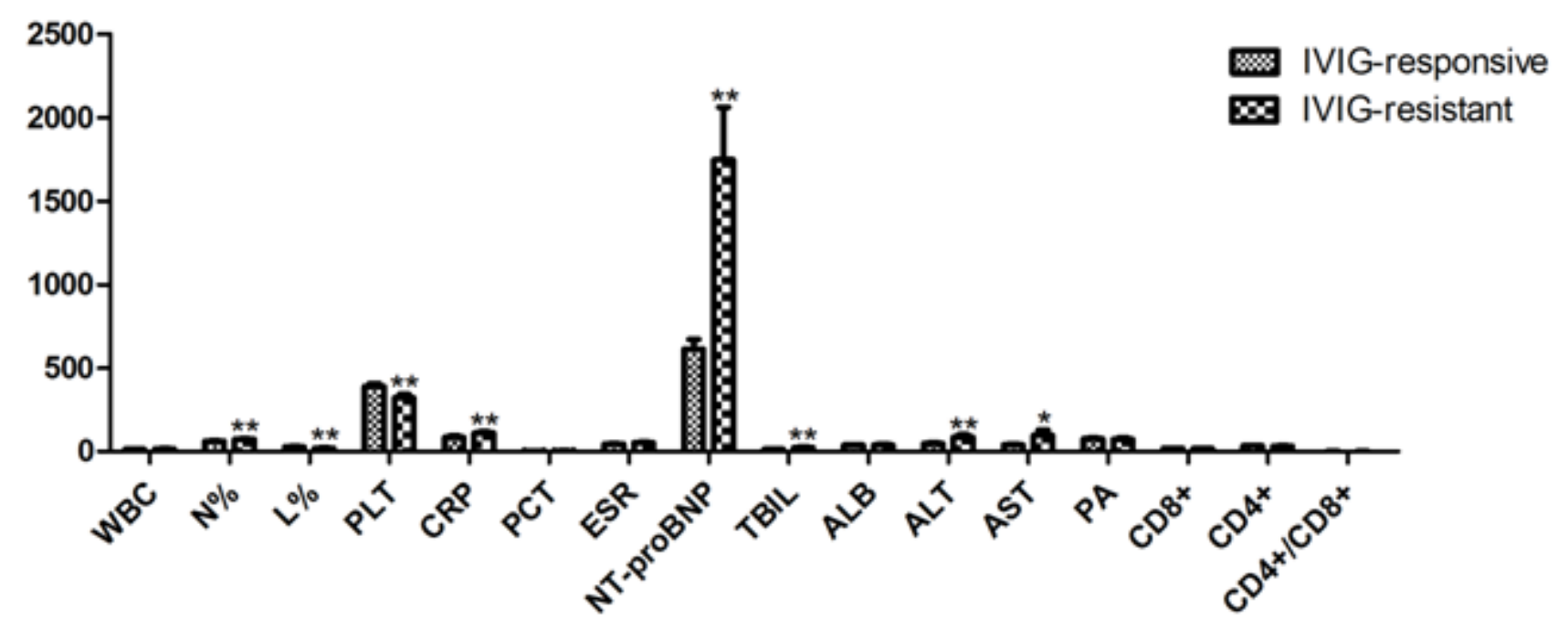

Figure 2

General analysis of whole blood isolated from KD patients between IVIG-responsive and IVIG-resistant patients. ${ }^{*} P<0.05,{ }^{\star}{ }^{*}<<0.01$ : IVIG-responsive group VS IVIG-resistant group. 
A

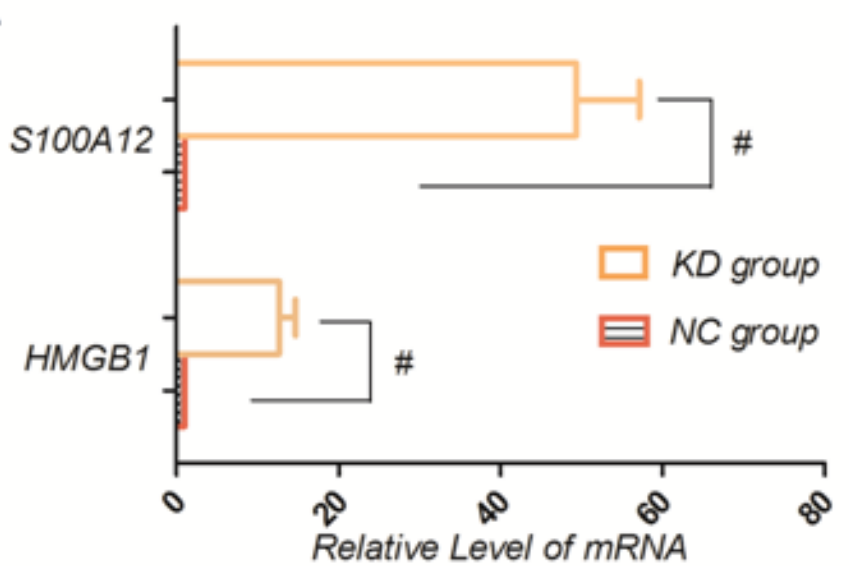

C

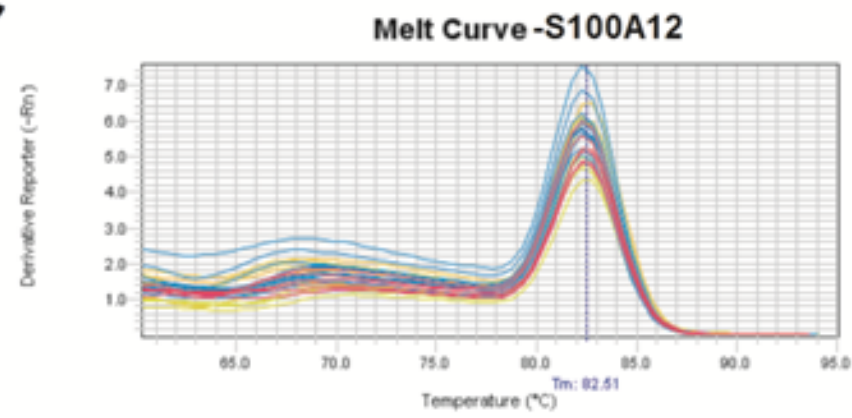

B

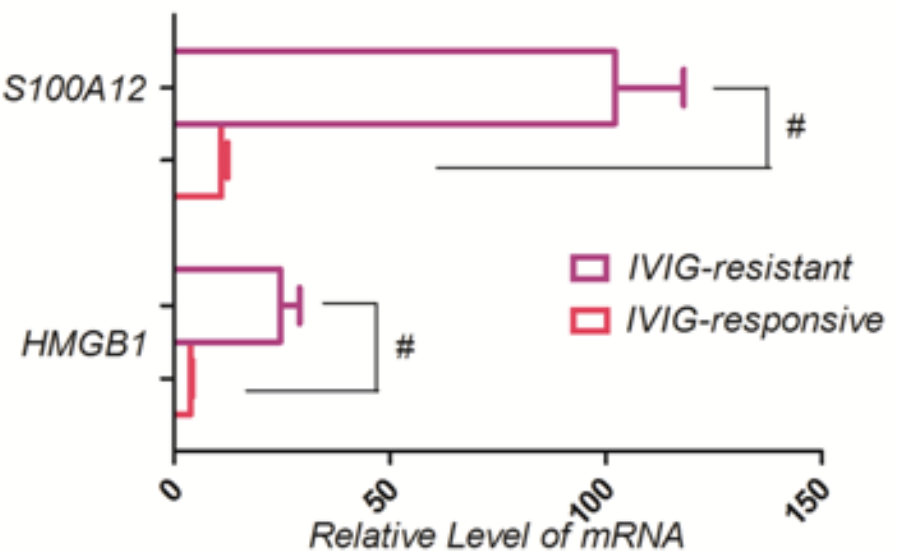

D

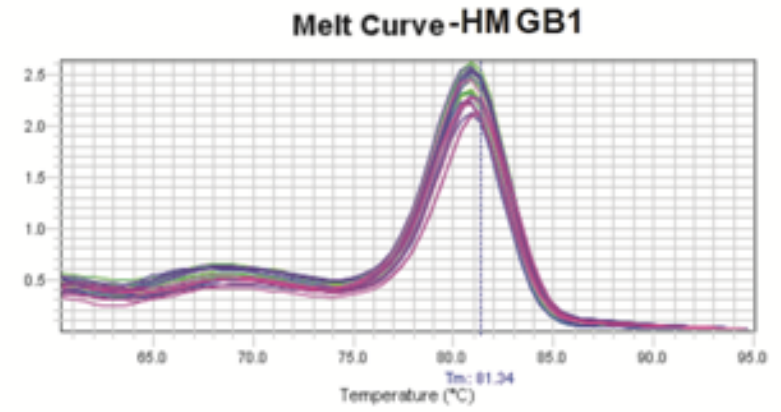

Figure 3

Data are shown as the mean \pm SD. ${ }^{*} P<0.05$ NC groupVS KD group(A); The melt curve of $S 100 A 12(C)$ and HMGB1(D). *P< 0.05 IVIG-responsive group VS IVIG-resistant group(B).

A

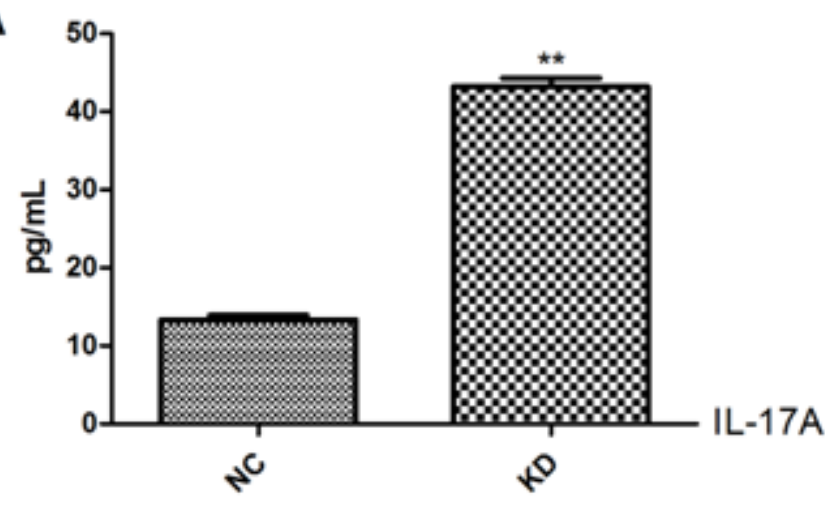

B



Figure 4 
serum IL-17A measurements by ELISA. Data are shown as the mean \pm SD. ${ }^{*} \mathrm{P}<0.01 \mathrm{NC}$ group VS KD group; ${ }^{\star *} P<0.01$ IVIG-responsive group VS IVIG-resistant group.

\section{Supplementary Files}

This is a list of supplementary files associated with this preprint. Click to download.

- renamed24630.xls 